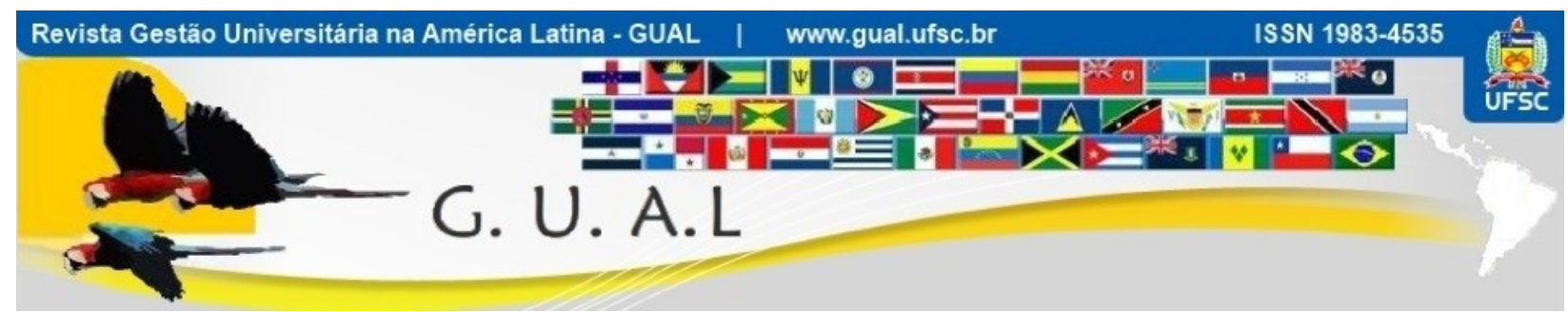

DOI: http://dx.doi.org/10.5007/1983-4535.2018v11n3p305

\title{
AVALIAÇÃO DA QUALIDADE DOS SERVIÇOS DE EDUCAÇÃO A DISTÂNCIA NO ENSINO SUPERIOR: PROPOSTA E AVALIAÇÃO DE ESCALA DE MENSURAÇÃO
}

\author{
EVALUATION OF THE QUALITY OF DISTANCE EDUCATION SERVICES IN \\ HIGHER EDUCATION: PROPOSAL AND EVALUATION OF MEASUREMENT \\ SCALE
}

\author{
Aléssio Bessa Sarquis, Doutor \\ Universidade do Sul de Santa Catarina - UNISUL \\ alessio.sarquis@,gmail.com \\ João Coelho Soares, Mestre \\ Universidade do Sul de Santa Catarina - UNISUL \\ joaocsoares88@gmail.com \\ Carolina Bez Batti Hübbe, Graduada \\ Universidade do Sul de Santa Catarina - UNISUL \\ carolina.hubbe@unisul.br \\ Vilmar Gonçalves, Doutor \\ Universidade Federal de Pelotas - UFpel \\ vtondolo@gmail.com \\ Rosana da Rosa Portella Tondolo, Doutora \\ Universidade Federal de Pelotas - UFpel \\ rosanatondolo@gmail.com
}

Recebido em 07/fevereiro/2018

Aprovado em 05/agosto/2018

Sistema de Avaliação: Double Blind Review

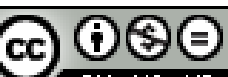

Esta obra está sob uma Licença Creative Commons Atribuição-Uso. 


\title{
RESUMO
}

No ensino superior privado, um dos grandes desafios é mensurar a qualidade percebida dos serviços de educação a distância. Com base na literatura de marketing, o artigo analisou as dimensões da qualidade percebida do serviço de educação à distância de uma instituição comunitária de ensino superior, na perspectiva do estudante, propondo e avaliando uma escala de mensuração. Trata-se de pesquisa quantitativa, descritiva, com levantamento de campo e estudo de caso. A coleta de dados envolveu questionário auto administrado e a participação de 459 estudantes. Os resultados indicam que a escala de mensuração proposta tem confiabilidade e validade, podendo ser utilizada na mensuração da qualidade percebida dos serviços, na perspectiva do estudante; que os serviços da instituição de ensino superior analisada têm qualidade elevada nos atributos relevância das disciplinas, conhecimento dos instrutores e atendimento da coordenação; que os atributos dos serviços da IES com menor qualidade percebida são incentivo à pesquisa e estímulo à criticidade dos estudantes. O estudo contribuiu para a identificação de dimensões e variáveis envolvidas na mensuração da qualidade percebida de serviços EAD no contex to do ensino superior.

Palavras chave: Marketing. Qualidade de Serviços. Qualidade Percebida. Educação à Distância.

\begin{abstract}
In private higher education, one of the major challenges is to measure the perceived quality of distance education services. Based on the marketing literature, the article analyzed the dimensions of the perceived quality of the distance education service of a community institution of higher education, from the perspective of the student, proposing and evaluating a measurement scale. It is a quantitative, descriptive research, survey and case study. Data collection involved a self-administered questionnaire and the participation of 459 students. The results indicate that the proposed measurement scale has reliability and validity, and can be used to measure the perceived quality of services, from the perspective of the student; that the services of the institution of higher education analyzed have high quality in the attributes relevance of the disciplines, knowledge of the instructors and attendance of the coordination; that the attributes of IES services with lower perceived quality are an incentive to research and stimulate the students' criticality. The study contributed to the identification of dimensions and variables involved in the measurement of the perceived quality of EAD services in the context of higher education.
\end{abstract}

Keywords: Marketing; Quality of Services. Perceived Quality. Distance Education. 


\section{AVALIAÇÃO DA QUALIDADE DOS SERVIÇOS DE EDUCAÇÃO A DISTÂNCIA NO ENSINO \\ SUPERIOR: PROPOSTA E AVALIAÇÃO DE ESCALA DE MENSURAÇÃO \\ DOI: http://dx.doi.org/10.5007/1983-4535.2018v11n3p305}

\section{INTRODUÇÃO}

O ensino a distância (EAD) tem se popularizado no Brasil. Conforme o Instituto Nacional de Estudos e Pesquisas Educacionais Anísio Teixeira, havia em 2012 cerca de 1.113.850 de estudantes e 1.329.407 de vagas (INEP, 2013). Em 2001, existiam apenas 13.967 estudantes e 6.856 vagas. Segundo o Censo EAD 2015, o Brasil possui mais de 5.048.912 estudantes nos cursos à distância (ABED, 2016), representando um crescimento de $30,5 \%$ sobre o ano anterior.

A educação a distância compreende a aprendizagem em local diferente de onde o ensino acontece (MOORE; KEARSLEY, 2007). Para De Souza et al. (2015) é a modalidade cuja mediação pedagógica ocorre pela utilização de tecnologias da informação e comunicação, com estudantes e professores desenvolvendo atividades em lugares e momentos diferentes. $\mathrm{O}$ ensino a distância possibilita estudar com menor custo e em conjunto com a educação presencial.

No Brasil, com o crescimento da educação a distância, aumentou também a concorrência entre as instituições de ensino privadas e a necessidade de avaliar a qualidade dos serviços. No ensino, a qualidade percebida afeta a satisfação dos estudantes e a competitividade da instituição (TOLENTINO; GONÇALVES FILHO; TOLENTINO; MONTEIRO, 2013), sendo utilizada como estratégia de marketing. Acredita-se, no entanto, que os serviços de educação à distância no Brasil carecem de melhorias (TOLENTINO et al, 2013; ABED, 2016) e que há incertezas sobre a sua eficácia (DE SOUZA et al., 2015). As instituições de ensino privadas precisam focalizar mais nos estudantes, mensurar melhor o desempenho dos serviços e assegurar que a qualidade atenda, efetivamente, as necessidades dos estudantes. Necessitam, também, conhecer melhor como os estudantes percebem a educação a distância e desenvolver estratégicas de marketing adequadas ao perfil dos estudantes (MANTOVANI; GOUVÊA; TAMASHIRO, 2015).

Na literatura de marketing, Zeithaml, Bitner e Gremler (2014) definiram a qualidade percebida como aquela mensurada a partir da comparação de expectativas e percepções do cliente. Assim, a qualidade percebida está associada a como o estudante percebe o valor do serviço (DALMONECH; GOULARTE; RAMOS; MONTE-MOR, 2016). Nos serviços EAD, a mensuração da qualidade percebida envolve coletar a opinião de estudantes sobre atributos do serviço, incluindo metodologia, professores e infraestrutura (SULTAN; WONG, 2013). Para isso, a instituição necessita compreender as dimensões (fatores) da qualidade percebida 


\section{AVALIAÇÃO DA QUALIDADE DOS SERVIÇOS DE EDUCAÇÃO A DISTÂNCIA NO ENSINO SUPERIOR: PROPOSTA E AVALIAÇÃO DE ESCALA DE MENSURAÇÃO \\ DOI: http://dx.doi.org/10.5007/1983-4535.2018v11n3p305}

dos serviços e os respectivos atributos (variáveis) avaliados pelos estudantes (MILAN; DE TONI; MAIOLI, 2013).

Acredita-se, também, que há necessidade de aprofundamento nos estudos sobre escalas de mensuração da qualidade percebida dos serviços de educação à distância. No Brasil, trata-se de uma modalidade de ensino relativamente nova, complexa, que envolve diversas dimensões/variáveis (DE ANDRADE; DE ANDRADE; MISAEL, 2015). Além disso, há poucas pesquisas no país sobre qualidade percebida no contexto da educação a distância e considerando a perspectiva do estudante. Consulta realizada nas bases de dados Google Acadêmico, SPELL da Anpad e Portal de Periódicos CAPES/MEC revelou a existência de poucos estudos científicos na área de marketing sobre o tema, e a necessidade de ampliar os estudos sobre escalas de mensuração no contexto da EAD.

Diante desse contexto, este estudo busca responder a seguinte questão de pesquisa: Quais são as dimensões da qualidade percebida do serviço de educação à distância na instituição comunitária de ensino superior, segundo a perspectiva do estudante? Com isso, o objetivo geral do estudo é analisar as dimensões da qualidade percebida por estudantes em relação aos serviços de educação a distância no ensino superior, em nível de graduação, em uma instituição de ensino comunitária, propondo e avaliando uma escala de mensuração.

\section{FUNDAMENTAÇÃO TEÓRICA}

\subsection{QUALIDADE PERCEBIDA DE SERVIÇOS}

O termo qualidade tem sido aplicado em diferentes contextos e depende de fatores que, quando alterados, modificam a percepção do avaliador (SONEHARA, 2011). No entanto, acredita-se que a qualidade é influenciada pelas características e capacidades do serviço em satisfazer parâmetros relevantes na perspectiva do cliente (MILAN; BRENTANO; DE TONI, 2008) e que a qualidade percebida afeta o comportamento pós-compra dos clientes (CLEMENTE-RICOLFE, 2016).

Zeithaml et al., (2014) definem a qualidade percebida de serviços como aquela mensurada a partir da comparação de expectativas e percepções do cliente. Simić e Carapić (2008) afirmam, por sua vez, que a qualidade percebida está associada a como o cliente percebe o valor do serviço e é influenciada pela satisfação com o serviço. No âmbito deste artigo, compreende-se a qualidade percebida do serviço de educação à distância como o resultado da qualidade mensurada segundo a percepção do estudante de EAD. 


\section{AVALIAÇÃO DA QUALIDADE DOS SERVIÇOS DE EDUCAÇÃO A DISTÂNCIA NO ENSINO SUPERIOR: PROPOSTA E AVALIAÇÃO DE ESCALA DE MENSURAÇÃO \\ DOI: http://dx.doi.org/10.5007/1983-4535.2018v11n3p305}

$\mathrm{Na}$ prestação de serviços, sabe-se que a qualidade pode ser mensurada com base na opinião do cliente ou por meio da observação do processo de prestação do serviço (ZEITHAML; BERRY; PARASURAMAN, 1988). Gruber, Fuß, Voss e Gläser-Zikuda (2010) destacam que o conceito de qualidade depende do ponto de vista das pessoas interessadas no serviço e é influenciado pela necessidade individual dos envolvidos. Como cada pessoa possui percepção particular sobre suas necessidades/expectativas, a empresa necessita mensurar a qualidade das características que influenciam na qualidade percebida e que afetam a satisfação dos clientes.

Nos últimos anos, os estudos sobre a qualidade no contexto de serviços enriqueceram a teoria de marketing. Há vários estudos realizados sobre o tema, que ajudaram a ampliar as pesquisas no campo do marketing de serviços. Os resultados de alguns estudos indicam que a qualidade percebida varia conforme a perspectiva do avaliador (ex: cliente, funcionários, gestores) e que as organizações necessitam aprimorar a qualidade dos serviços (RADONS; TORRES; CERETTA, 2013). Sabe-se, também, que o cliente tende a manter relacionamento apenas com organizações que oferecem qualidade e que satisfazem suas necessidades (BARRETO; DOS SANTOS; DA SILVA; GOMES; DE MENEZES, 2012), e que há qualidade percebida no serviço quando a organização consegue atender ou superar as expectativas do cliente (VIEIRA; BARBOSA, 2011).

\subsection{AVALIAÇÃO DA QUALIDADE NA EDUCAÇÃO}

Conforme Netto e Giraffa (2010), a preocupação com a qualidade nos serviços de educação se intensificou nos últimos anos e ampliou o interesse pela mensuração da qualidade. Sabe-se, também, que investimentos em qualidade na educação gera benefícios tanto para a instituição de ensino como para os estudantes (BERGAMO; GIULIANI; GALLI, 2011). Na educação, a avaliação da qualidade é uma tarefa difícil e pode variar conforme o propósito da avaliação, a perspectiva do avaliador e método de avaliação (GÓMEZ-REY; BARBERA; FERNÁNDEZ-NAVARRO, 2016). Acredita-se, por exemplo, que a qualidade percebida tende a variar quando mensurada segundo a perspectiva de gestores ou funcionários (GRUBER et al., 2010). Que a qualidade percebida pode variar quando mensurada por meio de avaliações pessoais, observação, feedback, pesquisas com questionários ou pela evolução de perdas/reclamações (SONEHARA, 2011). Além disso, no setor de educação, o serviço é predominantemente intangível (difícil de ser tocado, visto e estocado) e precisa ser, também, 


\section{AVALIAÇÃO DA QUALIDADE DOS SERVIÇOS DE EDUCAÇÃO A DISTÂNCIA NO ENSINO \\ SUPERIOR: PROPOSTA E AVALIAÇÃO DE ESCALA DE MENSURAÇÃO \\ DOI: http://dx.doi.org/10.5007/1983-4535.2018v11n3p305}

avaliado a partir de indicadores subjetivos e na perspectiva do usuário (ZEITHAML et al., 1988).

$\mathrm{Na}$ educação a distância, a instituição de ensino necessita mensurar a qualidade percebida de vários aspectos dos serviços, incluindo ação pedagógica, tutor presencial, metodologia do curso, conteúdo lecionado, infraestrutura e material didático (LOURENÇO; KNOP, 2011). Há, também, a necessidade de se avaliar periodicamente e considerando as normas reguladoras de órgãos governamentais. Uma das formas de avaliar a qualidade percebida na educação é mensurar a opinião dos estudantes sobre a qualidade dos serviços. Ao consumir tais serviços, o estudante tende a perceber a qualidade da instituição e avaliar a satisfação pessoal com os serviços oferecidos (ERGANG et al., 2012; PRIETO et al., 2018). O estudo de Duque Oliva, Cervera Taulet e Rodríguez Romero (2006) apontou que o paradigma da desconfirmação das expectativas serve para a avaliação da qualidade percebida de serviços pela Internet. Dentre os estudos relevantes sobre o tema, o trabalho de Alves (2003) destaca-se pela avaliação da qualidade de serviços de educação no contexto da universidade e na percepção dos estudantes. Os resultados do estudo indicam que a percepção dos estudantes varia conforme o contexto da universidade (se pública ou privada), e que a origem do estudante influencia na percepção sobre a qualidade. Já Venturini, Pereira, Vieira e Milach (2008) analisaram, mediante modelagem de equações estruturais, a satisfação de estudantes com a qualidade do curso de Ciências Contábeis (231 participantes), e revelou que a satisfação com a qualidade é influenciada pelo envolvimento do professor e pelo interesse do estudante, aspectos que dependem da organização do curso e da forma de interação entre professor e estudantes.

Senthilkumar e Arulraj (2011) desenvolveram escala de mensuração da qualidade percebida para instituições de ensino superior e concluíram que há necessidade de mensurar a metodologia de ensino, os recursos físicos disponíveis, as disciplinas ofertadas e a empregabilidade dos estudantes. Já a pesquisa de Douglas, Douglas e Barnes (2006) avaliou a satisfação dos estudantes com a qualidade dos serviços em uma universidade no Reino Unido e concluíram que determinantes da satisfação são atributos vinculados à qualidade do ensino e à aprendizagem; e que os estudantes estão dispostos a tolerar desacertos em outras áreas da instituição, se houver satisfação com a qualidade do ensino. 


\section{AVALIAÇÃO DA QUALIDADE DOS SERVIÇOS DE EDUCAÇÃO A DISTÂNCIA NO ENSINO SUPERIOR: PROPOSTA E AVALIAÇÃO DE ESCALA DE MENSURAÇÃO DOI: http://dx.doi.org/10.5007/1983-4535.2018v11n3p305}

\subsection{DIMENSÕES DA QUALIDADE NO EAD}

Os avanços tecnológicos possibilitaram a melhoria do ensino a distância e o crescimento da oferta de cursos (DOS SANTOS et al., 2015). Com isso, a literatura acadêmica tem dado maior atenção à gestão da qualidade dos serviços e à mensuração da qualidade percebida (AGUADED; MEDINA-SALGUERO, 2015; GÓMEZ-REY at al., 2016). No entanto, como apontado por Torres Moraga (2010), a qualidade percebida em serviços tende a ser um constructo multidimensional e cuja escala de mensuração carece de diferenças em relação à outras escalas propostas, conforme a natureza do serviço e organização. No Brasil, há alguns estudos interessantes sobre qualidade em serviços de educação a distância, com diferentes propósitos, métodos de pesquisa e dimensões/variáveis mensuradas.

O estudo de Tolentino et al. (2013), por exemplo, buscou mensurar a qualidade percebida na educação a distância, a partir de escala de satisfação projetada para modalidade presencial. No estudo, a qualidade foi mensurada por meio das dimensões: qualidade de ensino e qualidade dos serviços administrativos. A qualidade de ensino foi mensurada pelas variáveis: qualidade dos professores, qualidade da estrutura, qualidade das disciplinas, qualidade da coordenação e qualidade do curso. Já a qualidade dos serviços abrangeu aspectos da estrutura de apoio, incluindo setor de ensino, tesouraria, biblioteca, espaço de convivência e laboratórios.

Mantovani et al. (2015) avaliaram a qualidade percebida de serviços de EAD sob a perspectiva de estudantes, via pesquisa quantitativa, descritiva e levantamento de campo. Foram mensuradas 28 variáveis, agrupadas em 6 dimensões, a partir da escala SERVQUAL adaptada, a saber: conteúdo do website, confiabilidade, presteza, empatia, segurança e qualidade geral. A dimensão conteúdo do website aborda aspectos tangíveis do curso, incluindo infraestrutura do ambiente virtual e qualidade do material didático; a confiabilidade foca na capacidade do docente em inspirar confiança nos estudantes; a presteza trata da disposição do docente em ajudar o estudante; a empatia aborda a capacidade do docente em estabelecer relacionamento com o estudante; a segurança aborda o nível de conhecimento e domínio do docente.

Orosco, Puglia e Duarte (2014) avaliaram a qualidade de serviços de educação na modalidade semipresencial, por meio de pesquisa descritiva, transversal e quantitativa. A qualidade percebida foi mensurada por variáveis de entradas (inputs), de processo e de 


\section{AVALIAÇÃO DA QUALIDADE DOS SERVIÇOS DE EDUCAÇÃO A DISTÂNCIA NO ENSINO SUPERIOR: PROPOSTA E AVALIAÇÃO DE ESCALA DE MENSURAÇÃO \\ DOI: http://dx.doi.org/10.5007/1983-4535.2018v11n3p305}

resultados dos serviços. As variáveis de entradas correspondem aos aspectos ligados aos gastos com educação, investimento em tecnologia e formação dos docentes. Já as variáveis de processo fazem alusão ao contexto pedagógico e organizacional, e incluem aspectos da participação dos estudantes no processo. Enquanto as variáveis de resultados incluem aspectos do desempenho dos estudantes e da instituição no processo de aprendizagem.

O estudo de De Souza et al. (2015) avaliou a qualidade percebida de serviços de educação a distância sob a perspectiva de estudantes egressos. O questionário foi estruturado segundo o modelo de avaliação de reação (satisfação), expressando o contentamento do estudante com a aprendizagem e resultados obtidos. As 52 variáveis mensuradas foram agrupadas em três dimensões: auto avaliação (22 variáveis), desempenho do tutor (11) e programa do curso (19).

Veiga, Moura, Gonçalves e Barbosa (1998) avaliaram a qualidade percebida de serviços de educação a distância por questionário com escala de três postos ("abaixo do esperado", "igual ao esperado" e "acima do esperado") e com base na opinião de estudantes. As 22 questões da escala Servqual foram adaptadas para medir a qualidade do serviço de EAD, nas dimensões: tangíveis, confiabilidade, presteza, segurança e empatia. Foram, também, incluídas questões sobre qualidade geral do curso e valor obtido, e informações adicionais como: o estudante terminou o curso, quais os motivos da desistência e razões para fazer o curso.

Por fim, o estudo de Pinto, Laurino e Lunardi (2015) envolveu a construção, validação e análise de instrumento de mensuração da qualidade de serviços de educação a distância, utilizando escala de concordância e a partir da opinião de estudantes. O instrumento de mensuração contém as dimensões: estrutura e organização do curso; navegação e usabilidade; interação dos estudantes; estrutura física do polo; recursos humanos; estrutura e conteúdo das disciplinas; tutoria a distância; professores. No final, o questionário contém questões abertas sobre aspectos positivos e negativos do curso.

\section{ASPECTOS METODOLÓGICOS}

Este estudo compreende pesquisa quantitativa, descritiva, na forma de levantamento de campo e estudo de caso. A pesquisa quantitativa envolve técnicas de coleta estruturadas e técnicas estatísticas de análise. Trata-se de pesquisa quantitativo-descritiva porque buscou dimensionar características e opiniões da população-alvo do estudo, com auxílio de técnica de 


\section{AVALIAÇÃO DA QUALIDADE DOS SERVIÇOS DE EDUCAÇÃO A DISTÂNCIA NO ENSINO SUPERIOR: PROPOSTA E AVALIAÇÃO DE ESCALA DE MENSURAÇÃO DOI: http://dx.doi.org/10.5007/1983-4535.2018v11n3p305}

coleta padronizada. A população do estudo compreende estudantes ativos (matriculados) em cursos de graduação, pós-graduação e extensão da ABSVirtual (nome fíctício), que frequentaram pelo menos uma disciplina de EAD até 25/10/2016, constituindo o total 5.837 estudantes. O processo de amostra foi não probabilístico, com seleção por conveniência e a amostra foi constituída por aqueles que aceitaram participar do estudo. O tamanho da amostra é 459 respondentes, representando um erro amostral de 4,39\% e intervalo de confiança de $95 \%$.

O método de coleta dos dados envolveu a aplicação de questionário auto administrado, disponibilizado na plataforma eletrônica Google Docs, no período de 19/10/16 a 26/10/16. O questionário foi elaborado em escala tipo Likert de 7 pontos (onde $7=$ "Concordo totalmente", 1 = "Discordo totalmente" e 4 = "Nem concordo, nem discordo"), e constituído por 6 perguntas sobre perfil dos respondentes e 35 afirmações distribuídas em 5 dimensões/fatores, conforme a Tabela 1. O questionário foi, previamente, validado pela equipe de gestores da ABSVirtual (validação de face) e, depois, pré-testado com 20 estudantes de EAD.

Tabela 1 Dimensões e variáveis presentes no questionário

\begin{tabular}{|c|l|c|c|}
\hline Dimensão & \multicolumn{1}{|c|}{ Variáveis } & \multicolumn{1}{|c|}{ Fonte } \\
\hline $\begin{array}{c}\text { Metodologia } \\
\text { de ensino }\end{array}$ & $\begin{array}{l}\text { Metodologia tem qualidade; metodologia dá segurança ao estudante; } \\
\text { metodologia incentiva a pesquisa; orientações sobre metodologia são } \\
\text { claras; disciplinas estão integradas; disciplinas são importantes; disciplinas } \\
\text { são compatíveis com carga horária; atividades contribuem para } \\
\text { aprendizagem; cronograma é adequado; avaliação é coerente com } \\
\text { conteúdo lecionado. }\end{array}$ & $\begin{array}{l}\text { Tolentino et al. } \\
\text { (2013); Orosco et } \\
\text { al. (2014) }\end{array}$ \\
\hline $\begin{array}{c}\text { Material } \\
\text { didático }\end{array}$ & $\begin{array}{l}\text { Material didático tem conteúdo relevante; material tem referências } \\
\text { atualizadas; material tem linguagem clara; midiateca oferece materiais de } \\
\text { qualidade. }\end{array}$ & $\begin{array}{l}\text { Mantovani et al. } \\
\text { (2015); Orosco et } \\
\text { al. (2014) }\end{array}$ \\
\hline Instrutores & $\begin{array}{l}\text { Instrutores têm conhecimento; instrutores são acessíveis; instrutores estão } \\
\text { preocupados em ajudar; instrutores atendem rapidamente; instrutores são } \\
\text { eficientes; instrutores são justos na avaliação; instrutores despertam o o } \\
\text { interesse; instrutores estimulam a criticidade; instrutores valorizam a a } \\
\text { participação; instrutores cumprem o que prometem. }\end{array}$ & $\begin{array}{l}\text { Mantovani et al. } \\
\text { (2015); Tolentino } \\
\text { et al. (2013) }\end{array}$ \\
\hline Estrutura & $\begin{array}{l}\text { Ferramentas do ambiente virtual tem qualidade; recursos multimídia têm } \\
\text { qualidade; instalações do polo presencial têm qualidade; suporte técnico } \\
\text { tem qualidade; apoio administrativo tem qualidade; biblioteca tem } \\
\text { qualidade. }\end{array}$ & $\begin{array}{l}\text { Mantovani et al. } \\
\text { (2015); Tolentino } \\
\text { et al. (2013) }\end{array}$ \\
\hline Coordenação & $\begin{array}{l}\text { Coordenação do curso é atencioso; coordenação é eficiente; coordenação é } \\
\text { acessível; coordenação responde às solicitações em tempo hábil. }\end{array}$ & $\begin{array}{l}\text { Tolentino } \text { et al. } \\
\text { (2013); Pinto et al. } \\
\text { (2015) }\end{array}$ \\
\hline
\end{tabular}




\section{AVALIAÇÃO DA QUALIDADE DOS SERVIÇOS DE EDUCAÇÃO A DISTÂNCIA NO ENSINO SUPERIOR: PROPOSTA E AVALIAÇÃO DE ESCALA DE MENSURAÇÃO DOI: http://dx.doi.org/10.5007/1983-4535.2018v11n3p305}

No método de análise, os questionários preenchidos foram verificados, os dados coletados tabulados e as análises feitas pelo Statistical Package for the Social Sciences. Na análise descritiva, utilizou-se as técnicas média, desvio padrão, frequência absoluta e frequência relativa. Na avaliação da escala, a confiabilidade foi verificada pela técnica Alpha de Cronbach, e a validação da escala pela técnica Análise Fatorial Exploratória Multivariada. Conforme Hair et al. (2010), a análise fatorial possibilita reduzir grande quantidade de variáveis a um número pequeno de fatores e analisar padrões de relações multidimensionais de variáveis, identificando as variáveis que devem ser agrupadas por fator/dimensão. Neste estudo, essa técnica estatística foi utilizada para avaliar a validade da escala proposta para mensuração da qualidade de serviços de EAD.

\section{APRESENTAÇÃO E ANÁLISE DOS RESULTADOS}

\subsection{APRESENTAÇÃO DO CASO - ABSVIRTUAL}

A ABSVirtual é instituição educacional privada orientada para a produção, desenvolvimento e difusão do conhecimento, por intermédio da pesquisa, ensino e extensão, em diferentes áreas de conhecimento, e nas modalidades presencial e a distância. Em 2002, a universidade foi credenciada pelo Ministério da Educação no Brasil para ofertar cursos de pós-graduação lato sensu pela modalidade de educação a distância. No ano seguinte, obteve o credenciamento para ofertar, também, cursos de graduação e sequenciais a distância. Atualmente, a instituição possui mais de 100 polos presenciais, espalhados em diferentes estados do Brasil. A metodologia dos cursos baseia-se em quatro elementos: recursos didáticos (livro didático, multimídia e web conferência), sistema tutorial (coordenador de curso, professores e tutores), sistema de avaliação (avaliação da aprendizagem) e sistema de comunicação (diferentes canais de comunicação que integram tecnologias de comunicação e informação).

\subsection{RESULTADOS DA MENSURAÇÃO DA QUALIDADE PERCEBIDA}

Esta seção apresenta o perfil da amostra do estudo e os resultados da análise descritiva da qualidade dos serviços da ABSVirtual. Na análise dos resultados, os atributos avaliados com pontuação 7 foram considerados como qualidade elevada; com pontuação 5 e 6 , como qualidade moderada; com pontuação 1,2 e 3, como qualidade baixa. Os estudantes que 


\section{AVALIAÇÃO DA QUALIDADE DOS SERVIÇOS DE EDUCAÇÃO A DISTÂNCIA NO ENSINO SUPERIOR: PROPOSTA E AVALIAÇÃO DE ESCALA DE MENSURAÇÃO \\ DOI: http://dx.doi.org/10.5007/1983-4535.2018v11n3p305}

assinalaram pontuação 4 ("Nem concordo, nem discordo") foram considerados como indecisos.

Quanto ao perfil da amostra, cerca de 36,2\% dos respondentes têm idade entre 25 a 34 anos, sendo que a maior parte deles $(59,2 \%)$ é do sexo masculino e $41 \%$ são solteiros; $28,3 \%$ são casados com filho. Em relação à escolaridade, 60,7\% deles possuem ensino superior incompleto e predomina estudantes com renda média-mensal de 1 a 7 salários-mínimos (71,7\%). A maioria dos respondentes $(98,3 \%)$ são estudantes de cursos de graduação. Assim, avalia-se que o perfil da amostra é diversificado e representa o público-alvo do estudo.

Figura 1 Dimensão qualidade percebida da metodologia

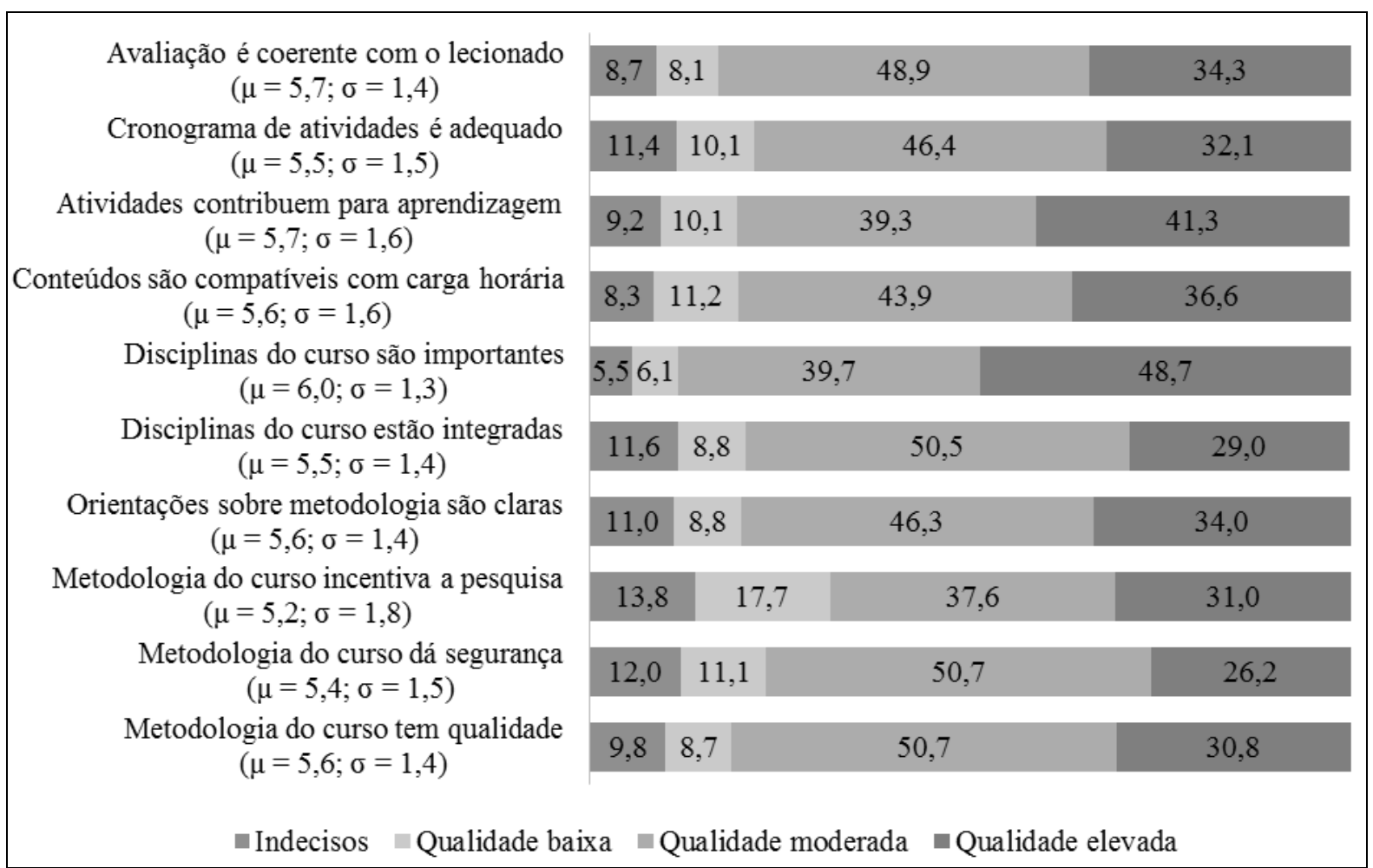

A Figura 1 apresenta os resultados da qualidade dos serviços de EAD na dimensão "Metodologia", que avaliou 10 atributos da metodologia do curso. A maioria dos estudantes avaliou que a metodologia da instituição tem qualidade. Os aspectos avaliados com qualidade elevada são: "disciplinas do curso são importantes" e "atividades do curso contribuem para aprendizagem". A variável "metodologia do curso incentiva a pesquisa" é a que apresenta maior quantidade de indecisos $(13,8 \%$ respondentes $)$, menor média de qualidade $(\mu=5,2)$ e maior desvio padrão nos dados coletados $(\sigma=1,8)$; já a variável "disciplinas do curso são 


\section{AVALIAÇÃO DA QUALIDADE DOS SERVIÇOS DE EDUCAÇÃO A DISTÂNCIA NO ENSINO SUPERIOR: PROPOSTA E AVALIAÇÃO DE ESCALA DE MENSURAÇÃO \\ DOI: http://dx.doi.org/10.5007/1983-4535.2018v11n3p305}

importantes" é a que apresenta a maior média de qualidade $(\mu=6)$ e menor desvio padrão $(\sigma$ $=1,3)$.

A Figura 2 contém os resultados da qualidade percebida da dimensão "Material Didático", que avaliou 5 atributos do material didático. Os resultados indicam que a maioria dos respondentes considera que o material didático da instituição tem qualidade moderada, em todos os atributos analisados. A variável "midiateca oferece materiais de qualidade" é a que apresenta maior quantidade de respondentes indecisos $(10,8 \%)$; as variáveis "material dos professores tem qualidade" e "material didático tem linguagem clara" apresentam baixo desvio padrão $(\sigma=1,2)$. Já a variável "livro didático tem referências atualizadas" apresenta a menor média de qualidade $(\mu=5,6)$ e desvio padrão mais elevado $(\sigma=1,4)$.

Figura 2 Dimensão qualidade percebida do material didático

\begin{tabular}{|c|c|c|c|}
\hline & & & \\
\hline $\begin{array}{l}\text { Midiateca oferece materiais de qualidade } \\
\qquad(\mu=5,7 ; \sigma=1,3)\end{array}$ & $10,86,6$ & 51,6 & 31,0 \\
\hline $\begin{array}{l}\text { Material dos professores tem qualidade } \\
\qquad(\mu=5,8 ; \sigma=1,2)\end{array}$ & $6,35,2$ & 53,9 & 34,5 \\
\hline $\begin{array}{l}\text { Material didático tem linguagem clara } \\
\qquad(\mu=5,9 ; \sigma=1,2)\end{array}$ & $7,94,4$ & 48,4 & 39,4 \\
\hline $\begin{array}{l}\text { Livro didático tem referências atualizadas } \\
\qquad(\mu=5,6 ; \sigma=1,4)\end{array}$ & $8,7 \quad 8,7$ & 51,1 & 31,4 \\
\hline $\begin{array}{l}\text { Livro didático tem conteúdo relevante } \\
\qquad(\mu=5,8 ; \sigma=1,3)\end{array}$ & $7,45,5$ & 50,2 & 36,9 \\
\hline Indecisos $\quad$ Qualidade baixa & Qualid & derada & evada \\
\hline
\end{tabular}

A Figura 3 mostra os resultados da qualidade da dimensão "Instrutores", que avaliou 10 atributos dos instrutores de cursos (professores e tutores). Os resultados indicam que 8 dos 10 atributos analisados têm qualidade moderada, na opinião da maioria dos respondentes. Os atributos considerados com qualidade elevada são: "instrutores cumprem o que prometem" e "instrutores têm conhecimento do conteúdo lecionado", obtendo esta última variável a maior média de qualidade $(\mu=6,1)$ e o menor desvio padrão $(\sigma=1,1)$. Na dimensão "Instrutores", a variável "instrutores estimulam a criticidade dos estudantes" é a que apresenta a menor média de qualidade $(5,2 \%)$, mas com desvio padrão elevado $(1,6)$, indicando maior dispersão na opinião dos respondentes. Há, também, diversas variáveis com elevada quantidade de indecisos, incluindo: "instrutores atendem rapidamente aos questionamentos dos estudantes" 


\section{AVALIAÇÃO DA QUALIDADE DOS SERVIÇOS DE EDUCAÇÃO A DISTÂNCIA NO ENSINO SUPERIOR: PROPOSTA E AVALIAÇÃO DE ESCALA DE MENSURAÇÃO \\ DOI: http://dx.doi.org/10.5007/1983-4535.2018v11n3p305}

(12,9\% de indecisos); "instrutores são eficientes nos questionamentos dos estudantes" $(11,1 \%)$; "instrutores estimulam a criticidade dos estudantes" (12,6\%); "instrutores valorizam a participação dos estudantes" (12,5\%).

Figura 3 Dimensão qualidade percebida dos instrutores

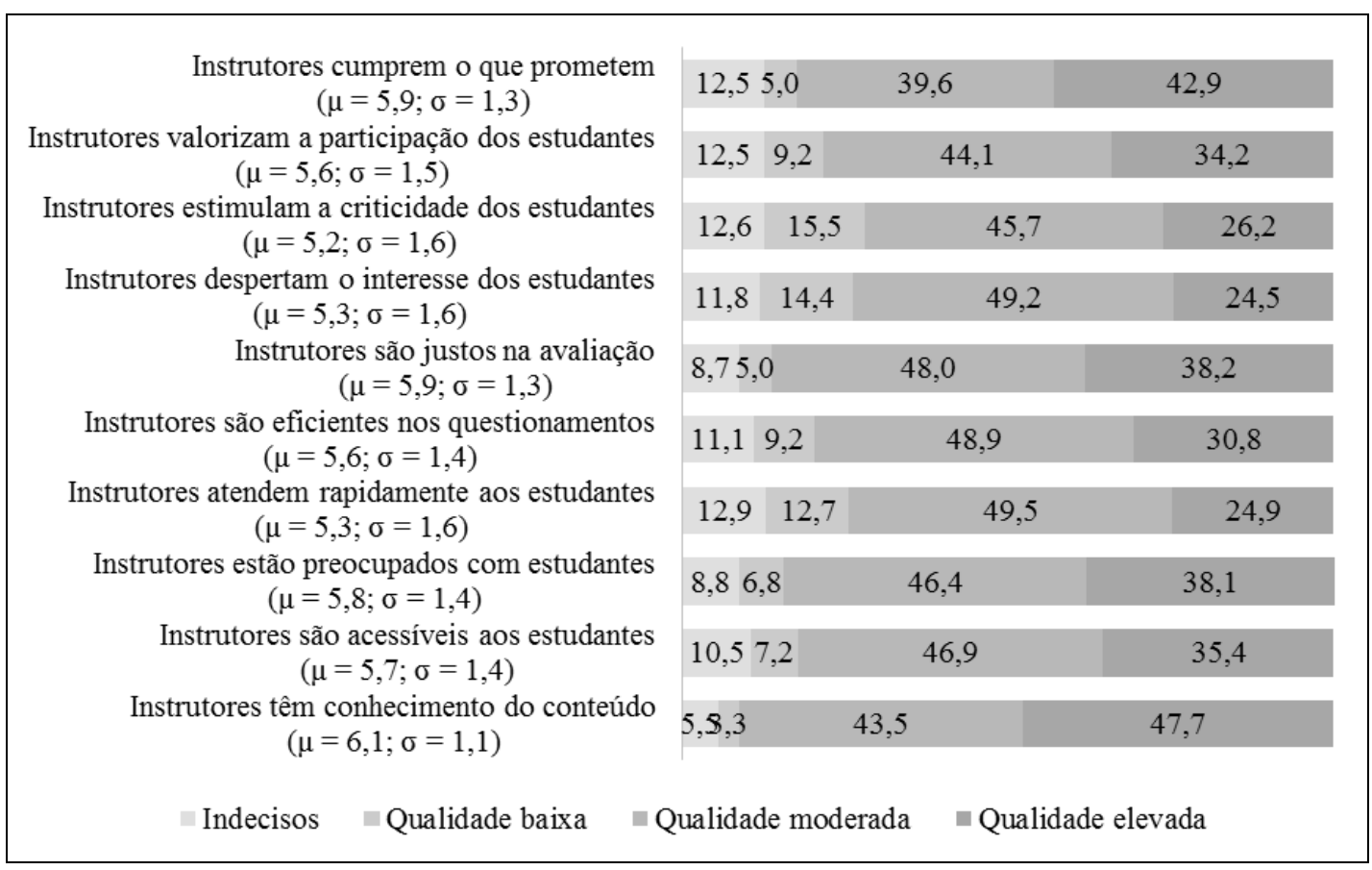

A Figura 4 apresenta os resultados da qualidade percebida da dimensão "Estrutura", que avaliou 6 atributos da estrutura dos cursos. Os resultados indicam que todos os atributos mensurados têm qualidade moderada, na opinião da maioria dos estudantes pesquisados.

Figura 4 Dimensão qualidade percebida da estrutura

\begin{tabular}{|c|c|c|c|}
\hline \multirow{2}{*}{$\begin{array}{l}\text { Biblioteca disponível tem qualidade } \\
(\mu=5,7 ; \sigma=1,4) \\
\text { Apoio administrativo tem qualidade } \\
(\mu=5,7 ; \sigma=1,5)\end{array}$} & $16,7 \quad 4,9$ & 41,7 & 36,6 \\
\hline & $11,3 \quad 7,1$ & 41,9 & 39,7 \\
\hline \multirow{3}{*}{$\begin{array}{l}\text { Suporte técnico do tutor tem qualidade } \\
\qquad(\mu=5,7 ; \sigma=1,3) \\
\text { Instalações físicas do polo presencial tem qualidade } \\
(\mu=5,4 ; \sigma=1,6) \\
\text { Recursos multimídia têm qualidade } \\
(\mu=5,7 ; \sigma=1,4)\end{array}$} & $11,5 \quad 6,2$ & 47,5 & 34,8 \\
\hline & $16,1 \quad 11,6$ & 39,7 & 32,6 \\
\hline & $11,8 \quad 7,0$ & 45,5 & 35,7 \\
\hline $\begin{array}{l}\text { Ambiente virtual tem tecnologia de qualidade } \\
\qquad(\mu=5,8 ; \sigma=1,3)\end{array}$ & $7,07,2$ & 45,6 & 40,2 \\
\hline Qualidade baixa & \multicolumn{2}{|c|}{ Qualidade moderada } & \\
\hline
\end{tabular}




\section{AVALIAÇÃO DA QUALIDADE DOS SERVIÇOS DE EDUCAÇÃO A DISTÂNCIA NO ENSINO SUPERIOR: PROPOSTA E AVALIAÇÃO DE ESCALA DE MENSURAÇÃO \\ DOI: http://dx.doi.org/10.5007/1983-4535.2018v11n3p305}

A variável com maior média de qualidade é “ambiente virtual tem ferramentas tecnológicas de qualidade”. Todas as demais variáveis apresentam percentual elevado de respondentes indecisos ou que avaliaram tais atributos com qualidade baixa (superior a 17\% dos respondentes). A variável "biblioteca tem qualidade" é a que apresenta maior quantidade de respondentes indecisos (16,7\%); a variável "instalações físicas do polo presencial tem qualidade" apresenta desvio padrão moderadamente elevado $(\sigma=1,6)$.

Por fim, a Figura 5 apresenta os resultados da qualidade da dimensão "Coordenação", aquela que avaliou 4 atributos dos serviços da coordenação do curso. Os resultados assinalam que essa é a dimensão com melhor qualidade. A maioria dos respondentes assinalou qualidade elevada para todas as variáveis mensuradas. A variável com maior média de qualidade $(\mu=6)$ é “coordenador é atencioso". No entanto, cabe observar que a variável "coordenador é eficiente na solução de problemas" apresenta elevada quantidade de respondentes indecisos ou que consideram a qualidade baixa $(18,2 \%)$. O desvio padrão de todas as variáveis mensuradas é baixo (inferior a $20 \%$ da escala).

Figura 5 Dimensão qualidade percebida da coordenação

Coordenador responde às solicitações em tempo hábil $(\mu=5,8 ; \sigma=1,4)$

Coordenador é acessível aos estudantes

$$
(\mu=5,9 ; \sigma=1,4)
$$

Coordenador é efíciente na solução de problemas $(\mu=5,9 ; \sigma=1,4)$

Coordenador é atencioso $(\mu=6,0 ; \sigma=1,3)$

\section{$11,66,2$} 39,3 42,9

$11,65,9 \quad 36,3 \quad 46,2$

\begin{tabular}{|c|c|}
\hline $12,95,3$ & 35,9 \\
\hline $10,54,2$ & 35,5 \\
\hline
\end{tabular}

Indecisos $\square$ Qualidade baixa $\square$ Qualidade moderada $\square$ Qualidade elevada

\subsection{RESULTADOS DA AVALIAÇÃO DA ESCALA DE MENSURAÇÃO APLICADA}

\subsubsection{Resultados da análise da confiabilidade}

O Alfa de Cronbach é uma técnica bastante utilizada para estimar a confiabilidade de escalas de mensuração (HAIR et al., 2010). Conforme Cronbach (1951), esse coeficiente permite analisar a confiabilidade de uma escala pela consistência interna do questionário, ou seja, pela magnitude da correlação dos itens mensurados. Como no questionário aplicado 


\section{AVALIAÇÃO DA QUALIDADE DOS SERVIÇOS DE EDUCAÇÃO A DISTÂNCIA NO ENSINO SUPERIOR: PROPOSTA E AVALIAÇÃO DE ESCALA DE MENSURAÇÃO \\ DOI: http://dx.doi.org/10.5007/1983-4535.2018v11n3p305}

todas as variáveis têm a mesma escala de mensuração (Likert 7 pontos), esse coeficiente pode ser calculado a partir da variância dos itens individuais e da variância da soma dos itens de cada respondente.

A Tabela 2 apresenta os resultados do cálculo do coeficiente Alfa das 5 dimensões do questionário aplicado (Metodologia EAD, Material didático EAD, Instrutores EAD, Estrutura EAD e Serviço da coordenação EAD), incluindo os resultados por variável e o coeficiente total da escala. Em geral, os resultados sugerem que a escala proposta tem confiabilidade para mensuração da qualidade percebida dos serviços de EAD. O coeficiente total da escala tem confiabilidade muito alta $(\alpha=0,968)$, segundo os critérios de Malhotra (2008); assim como nas dimensões Metodologia EAD $(\alpha=0,918)$, Instrutores EAD $(\alpha=0,950)$ e Serviço da coordenação $\operatorname{EAD}(\alpha=0,950)$. As demais dimensões da escala apresentam coeficientes de confiabilidade elevados: Material didático $\operatorname{EAD}(\alpha=0,871)$ e Estrutura $\operatorname{EAD}(\alpha=0,855)$. Efetuou-se também a análise de exclusão das variáveis com coeficientes alfa mais elevados. No entanto, os resultados dessa análise apontaram que as variações nos coeficientes alfa das dimensões foram pequenas, indicando a possibilidade de manutenção de todos as variáveis presentes na escala proposta. Na dimensão Metodologia EAD a redução foi de 0,918 para 0,907; em Instrutores de EAD foi de 0,950 para 0,946; em Serviços da coordenação EAD foi de 0,950 para 0,941 . Além disso, o resultado da análise por variável sugeriu a não necessidade de eliminar variáveis do questionário.

Tabela 2 Resultados de Alfa de Cronbach - por dimensão e variável da escala

\begin{tabular}{|c|c|c|}
\hline Dimensão & Variáveis/indicadores & Alfa $(\alpha)$ \\
\hline \multirow{10}{*}{ Metodologia ( 10 variáveis; $\alpha=0,918$ ) } & Metodologia tem qualidade & 0,903 \\
\hline & Metodologia dá segurança aos estudantes & 0,903 \\
\hline & Metodologia incentiva a pesquisa & 0,910 \\
\hline & Orientações sobre metodologia são claras & 0,911 \\
\hline & Disciplinas estão integradas & 0,909 \\
\hline & Disciplinas são importantes & 0,912 \\
\hline & Conteúdos são compatíveis com carga horária & 0,912 \\
\hline & $\begin{array}{l}\text { Atividades do curso contribuem para } \\
\text { aprendizagem }\end{array}$ & 0,907 \\
\hline & Cronograma de atividades é adequado & 0,914 \\
\hline & Avaliação é coerente com conteúdo lecionado & 0,911 \\
\hline \multirow{4}{*}{$\begin{array}{l}\text { Material didático (5 variáveis; } \\
\qquad \alpha=0,871)\end{array}$} & Material didático tem conteúdo relevante & 0,841 \\
\hline & Material tem referências atualizadas & 0,867 \\
\hline & Material tem linguagem clara & 0,843 \\
\hline & Material dos professores tem qualidade & 0,825 \\
\hline
\end{tabular}




\begin{tabular}{|c|c|c|}
\hline Dimensão & Variáveis/indicadores & Alfa $(\alpha)$ \\
\hline & Midiateca oferece materiais de qualidade & 0,844 \\
\hline \multirow{10}{*}{ Instrutores ( 10 variáveis; $\alpha=0,950$ ) } & $\begin{array}{l}\text { Instrutores têm conhecimento do conteúdo } \\
\text { lecionado }\end{array}$ & 0,949 \\
\hline & Instrutores são acessíveis aos estudantes & 0,943 \\
\hline & Instrutores estão preocupados com os estudantes & 0,942 \\
\hline & $\begin{array}{l}\text { Instrutores atendem rapidamente aos } \\
\text { questionamentos }\end{array}$ & 0,946 \\
\hline & Instrutores são eficientes nos questionamentos & 0,943 \\
\hline & Instrutores são justos na avaliação & 0,946 \\
\hline & Instrutores despertam o interesse dos estudantes & 0,942 \\
\hline & $\begin{array}{l}\text { Instrutores estimulam a criticidade dos } \\
\text { estudantes }\end{array}$ & 0,944 \\
\hline & $\begin{array}{l}\text { Instrutores valorizam a participação dos } \\
\text { estudantes }\end{array}$ & 0,945 \\
\hline & Instrutores cumprem o que prometem & 0,945 \\
\hline \multirow{6}{*}{ Estrutura ( 6 variáveis; $\alpha=0,855$} & $\begin{array}{l}\text { Ambiente virtual tem ferramentas tecnológicas } \\
\text { de qualidade }\end{array}$ & 0,819 \\
\hline & Recursos multimídia têm qualidade & 0,820 \\
\hline & $\begin{array}{l}\text { Instalações físicas do polo presencial tem } \\
\text { qualidade }\end{array}$ & 0,841 \\
\hline & Suporte técnico do tutor tem qualidade & 0,826 \\
\hline & Apoio administrativo tem qualidade & 0,835 \\
\hline & Biblioteca disponível tem qualidade & 0,843 \\
\hline \multirow{4}{*}{$\begin{array}{l}\text { Serviço da coordenação (4 variáveis; } \\
\qquad \alpha=0,950\end{array}$} & Coordenador é atencioso & 0,935 \\
\hline & Coordenador é eficiente na solução de problemas & 0,930 \\
\hline & Coordenador é acessível aos estudantes & 0,932 \\
\hline & $\begin{array}{l}\text { Coordenador responde às solicitações em tempo } \\
\text { hábil }\end{array}$ & 0,942 \\
\hline \multicolumn{2}{|c|}{ TOTAL DA ESCALA (35 itens/variáveis) } & 0,968 \\
\hline
\end{tabular}

Considerando que os valores de Alpha de Cronbach obtidos superam os valores mínimos recomendados por Malhotra (2008) e Hair et al. (2010), e que a escala de mensuração proposta apresenta coeficiente de confiabilidade em patamar semelhante ou superior a outros instrumentos de mensuração considerados aceitáveis (DA HORA; MONTEIRO; ARICA, 2010), avalia-se que a escala proposta pode ser utilizada na mensuração de qualidade percebida de serviços de EAD, na perspectiva de estudantes.

\subsubsection{Resultados da análise da validação da escala}

A validade da escala de mensuração proposta foi verificada com auxílio da técnica análise fatorial multivariada. Essa técnica é indicada para analisar padrões de relações 


\section{AVALIAÇÃO DA QUALIDADE DOS SERVIÇOS DE EDUCAÇÃO A DISTÂNCIA NO ENSINO SUPERIOR: PROPOSTA E AVALIAÇÃO DE ESCALA DE MENSURAÇÃO \\ DOI: http://dx.doi.org/10.5007/1983-4535.2018v11n3p305}

multidimensionais de variáveis, determinar o grau de explicação de cada dimensão e para identificar quais variáveis devem ser agrupadas por fator/dimensão. Neste trabalho, essa técnica foi utilizada para avaliar se os dados coletados satisfazem a estrutura esperada da escala de mensuração proposta, elaborada a partir de suporte teórico e resultados de estudos anteriores.

Alguns testes estatísticos foram realizados para verificar o grau de adequação dos dados coletados. No Kaiser-Meyer-Olkin, o resultado foi próximo de $1(0,954)$ indicando que a técnica é adequada no tratamento dos dados coletados. No teste de Bartlett, o resultado apontou valor Sig. menor que 0,01 , o que indica que os dados coletados são, também, adequados à técnica, e que as variáveis mensuradas estão correlacionadas significativamente.

A matriz de comunalidades das variáveis mensuradas foi analisada, e extraídos pelo método de análise dos componentes principais, um dos mais utilizados na análise de redução de dados (O'ROURKE; HATCHER, 2013). Na escala de mensuração proposta, os resultados de comunalidades nos fatores extraídos apontam que a porcentagem de variância de cada variável explicada é maior que $50 \%$ em todos os casos, indicando que na matriz de dados as variáveis têm correlações suficientes para viabilizar a aplicação da análise fatorial.

A Tabela 3 apresenta os resultados da matriz de componentes rotacionada, contendo as correlações entre variáveis e dimensões presentes na escala elaborada, e o poder explicativo. Os resultados indicaram que 5 fatores foram retidos (pelo método Análise dos Componentes Principais e rotacionados pelo método Varimax com Normalização de Kaiser), com autovalor superior a 1 (que é a soma de cargas fatoriais ao quadrado por fator e representa a quantidade de variância explicada). O poder explicativo total do modelo é $68,3 \%$ da variabilidade.

Tabela 3 Resultados da análise fatorial - matriz componente rotacionada

\begin{tabular}{|c|c|c|c|c|c|c|}
\hline \multirow{2}{*}{ Dimensão } & \multirow{2}{*}{ Variáveis } & \multicolumn{5}{|c|}{ Fatores } \\
\hline & & 1 & 2 & 3 & 4 & 5 \\
\hline \multirow{10}{*}{$\begin{array}{l}\frac{\pi}{0} 0 \\
\frac{0}{0} \\
\frac{0}{0} \\
\frac{0}{0} \\
\frac{0}{0}\end{array}$} & Metodologia do curso tem qualidade &, 761 & & & & \\
\hline & Metodologia do curso dá segurança aos & & & & & \\
\hline & estudantes & ,735 & & & & \\
\hline & Atividades do curso contribuem para & & & & & \\
\hline & aprendizagem & 690, & & & & \\
\hline & Metodologia do curso incentiva a pesquisa &, 683 & & & & \\
\hline & Disciplinas são importantes & 672 & & & & \\
\hline & Conteúdos são compatíveis com carga & & & & & \\
\hline & horária & ,659 & & & & \\
\hline & Disciplinas estão integradas & ,645 & & & & \\
\hline
\end{tabular}




\begin{tabular}{|c|c|c|c|c|c|c|}
\hline \multirow{3}{*}{ Dimensão } & \multirow{2}{*}{ Variáveis } & \multicolumn{5}{|c|}{ Fatores } \\
\hline & & 1 & 2 & 3 & 4 & 5 \\
\hline & $\begin{array}{l}\text { Avaliação é coerente com conteúdo } \\
\text { lecionado } \\
\text { Orientações sobre metodologia são claras } \\
\text { Cronograma de atividades é adequado }\end{array}$ & $\begin{array}{l}, 600 \\
, 592 \\
, 577\end{array}$ & & & & \\
\hline 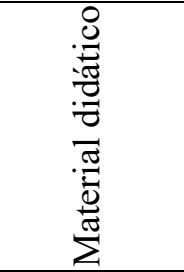 & $\begin{array}{l}\text { Material didático tem conteúdo relevante } \\
\text { Material tem linguagem clara } \\
\text { Material dos professores tem qualidade } \\
\text { Material tem referências atualizadas } \\
\text { Midiateca oferece materiais de qualidade }\end{array}$ & & & & & $\begin{array}{l}, 774 \\
, 716 \\
, 656 \\
, 628 \\
, 524\end{array}$ \\
\hline 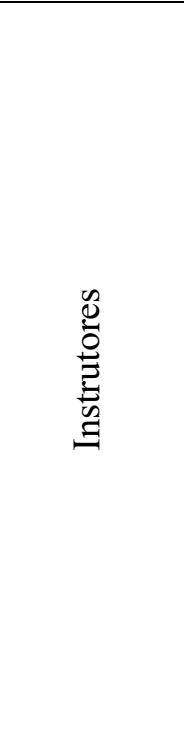 & $\begin{array}{l}\text { Instrutores estão preocupados com os } \\
\text { estudantes } \\
\text { Instrutores são eficientes } \\
\text { Instrutores são acessíveis aos estudantes } \\
\text { Instrutores atendem rapidamente } \\
\text { Instrutores são justos na avaliação } \\
\text { Instrutores cumprem o que prometem } \\
\text { Instrutores despertam o interesse dos } \\
\text { estudantes } \\
\text { Instrutores valorizam a participação dos } \\
\text { estudantes } \\
\text { Instrutores estimulam a criticidade dos } \\
\text { estudantes } \\
\text { Instrutores conhecem o conteúdo lecionado }\end{array}$ & & $\begin{array}{l}, 814 \\
, 782 \\
, 775 \\
, 750 \\
, 718 \\
, 710 \\
, 653 \\
, 623 \\
, 585 \\
, 488\end{array}$ & & & \\
\hline 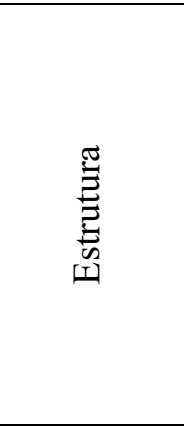 & $\begin{array}{l}\text { Recursos multimídia têm qualidade } \\
\text { Instalações físicas do polo presencial tem } \\
\text { qualidade } \\
\text { Ambiente virtual tem tecnológicas de } \\
\text { qualidade } \\
\text { Biblioteca disponível tem qualidade } \\
\text { Apoio administrativo tem qualidade } \\
\text { Suporte técnico do tutor tem qualidade }\end{array}$ & & & & $\begin{array}{r}, 708 \\
, 688 \\
, 639 \\
, 591 \\
, 564 \\
, 530 \\
\end{array}$ & \\
\hline 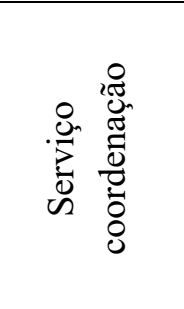 & $\begin{array}{l}\text { Coordenação é acessível aos estudantes } \\
\text { Coordenação responde às solicitações em } \\
\text { tempo hábil } \\
\text { Coordenação é eficiente na solução de } \\
\text { problemas } \\
\text { Coordenação é atenciosa }\end{array}$ & & & $\begin{array}{l}867 \\
, 849 \\
, 836 \\
, 825\end{array}$ & & \\
\hline TOTAL D & IIANCIA EXPLICADA $(\mathrm{em} \%)=68,3$ & 19,1 & 18,6 & 11,2 & 10,3 & 9,1 \\
\hline
\end{tabular}

Os resultados da análise fatorial mostram as variáveis agrupadas em 5 fatores, que representam dimensões da qualidade percebida do serviço de EAD. O fator 1, denominado 


\section{AVALIAÇÃO DA QUALIDADE DOS SERVIÇOS DE EDUCAÇÃO A DISTÂNCIA NO ENSINO SUPERIOR: PROPOSTA E AVALIAÇÃO DE ESCALA DE MENSURAÇÃO \\ DOI: http://dx.doi.org/10.5007/1983-4535.2018v11n3p305}

"Metodologia EAD", agrupa as 10 variáveis relacionadas à metodologia do curso de EAD (todas com carga fatorial superior a 0,5), principalmente as variáveis: metodologia do curso tem qualidade, metodologia do curso dá segurança aos estudantes, atividades do curso contribuem para aprendizagem, metodologia do curso incentiva a pesquisa, disciplinas do curso são importantes, conteúdo do curso é compatível com carga horária, disciplinas do curso estão integradas e avaliação da aprendizagem é coerente com o conteúdo lecionado. Essa dimensão tem poder explicativo de 19,1\%.

O fator 2, denominado "Instrutores de EAD", agrupa as 10 variáveis relacionadas à qualidade dos instrutores, incluindo as variáveis: instrutores estão preocupados com os estudantes, instrutores são eficientes no atendimento aos questionamentos dos estudantes, instrutores são acessíveis aos estudantes, instrutores atendem rapidamente aos questionamentos dos estudantes, instrutores são justos na avaliação da aprendizagem, instrutores cumprem o que prometem, instrutores despertam o interesse dos estudantes, instrutores valorizam a participação dos estudantes. O poder explicativo é de $18,6 \%$. O "Serviço da Coordenação EAD", fator 3, contém as 04 variáveis relacionadas à qualidade do serviço da coordenação do curso (todas com carga fatorial superior a 0,8), quais sejam: coordenação é acessível aos estudantes, coordenação responde às solicitações dos estudantes em tempo hábil, coordenação é eficiente na solução de problemas dos estudantes e coordenação é atenciosa com os estudantes. Poder explicativo de 11,2\%.

O fator 4, designado "Estrutura de EAD", agrupa as 06 variáveis relacionadas à qualidade da estrutura oferecida pelo curso (todas com carta fatorial superior a 0,5 ), incluindo principalmente as variáveis: recursos multimídia têm qualidade, instalações físicas do polo presencial tem qualidade e ambiente virtual tem ferramentas tecnológicas de qualidade. Poder explicativo de 10,3\%. Já o fator 5, denominado "Material Didático EAD”, reúne o conjunto de 05 variáveis relacionadas à qualidade do material didático (todas com carga fatorial superior a 0,5$)$, incluindo as variáveis: livro didático tem conteúdo relevante, material didático tem linguagem clara, material dos professores tem qualidade e livro didático tem referências atualizadas. Poder explicativo de 9,1\%.

Com isso, avalia-se que os resultados da análise fatorial confirmam a estrutura esperada da escala de mensuração proposta, elaborada a partir de suporte teórico e resultados de estudos anteriores. Tais resultados indicam que a escala de mensuração proposta possui 


\section{AVALIAÇÃO DA QUALIDADE DOS SERVIÇOS DE EDUCAÇÃO A DISTÂNCIA NO ENSINO SUPERIOR: PROPOSTA E AVALIAÇÃO DE ESCALA DE MENSURAÇÃO \\ DOI: http://dx.doi.org/10.5007/1983-4535.2018v11n3p305}

validade (validade fatorial) e que pode ser aplicada na mensuração da qualidade percebida do serviço de EAD, sob a perspectiva de estudantes.

\section{CONSIDERAÇÕES FINAIS}

O presente estudo buscou analisou as dimensões da qualidade percebida do serviço de educação à distância na instituição de ensino privada, na perspectiva do estudante brasileiro, propondo e avaliando uma escala de mensuração. Acredita-se que os objetivos do estudo foram atingidos. Uma escala de mensuração foi proposta com cinco dimensões: qualidade da metodologia do curso, qualidade do material didático, qualidade dos instrutores (professores e tutores), qualidade da infraestrutura e qualidade da coordenação.

A escala de mensuração proposta foi avaliada em termos de confiabilidade e validade. Os resultados indicam que a escala aplicada tem confiabilidade e validade, e pode ser utilizada na mensuração da qualidade percebida do serviço de EAD, na perspectiva de estudantes. Como recomendado por Lourenço e Knop (2011), o estudo elaborou escala de mensuração da qualidade percebida considerando ampla variedade de dimensões e variáveis envolvidas nos serviços EAD. Diferente do trabalho de Orosco et al. (2014), que avaliou a qualidade na modalidade semipresencial, o estudo avaliou a qualidade percebida considerando apenas a opinião de estudantes de EAD. Diferente dos estudos de Ergang et al. (2012) e Douglas et al. (2006), que avaliaram a partir da satisfação dos estudantes, o estudo mensurou diretamente a qualidade percebida dos serviços; e desenvolveu a escala de mensuração a partir de várias dimensões e variáveis recomendadas por outros estudos, diferenciando-se do trabalho de Tolentino et al. (2013), que mensurou a qualidade a partir de escala projetada para a modalidade presencial, e de Mantovani et al. (2015), que mensurou a qualidade a partir da escala SERVQUAL adaptada.

Como direcionamento para futuros estudos, sugere-se a replicação da escala proposta em outras instituições, com propósito de confirmar a confiabilidade e validade. Sugere-se, também, que a análise da validação seja avaliada pela técnica estatística Análise Fatorial Confirmatória (AFC), que permite testar em que medida as variáveis mensuradas são representativas das dimensões propostas. Sugere-se, ainda, que a qualidade percebida da educação a distância seja mensurada sob as perspectivas de professores e funcionários administrativos. 


\section{REFERÊNCIAS}

ABED. Censo EaD.br 2015: relatório da aprendizagem a distância no Brasil. Disponível em http://www.abed.org.br/site/pt/midiateca/censo_ead. Acesso em 20 agosto, 2016.

AGUADED, I.; MEDINA-SALGUERO, R. Criterios de calidad para la valoración y gestión de MOOC. Revista Iberoamericana de Educación a Distancia, v. 18, n. 2, 2015.

ALVES, G. Marketing en los servicios de educación: modelos de percepción de calidad. 119p. Dissertação (Mestrado em Marketing) - Universidad Complutense de Madrid, 2003.

BARRETO, E.; DOS SANTOS, R.; DA SILVA, L.; GOMES, M.; DE MENEZES, V. Aplicação do método Servqual na avaliação da satisfação de clientes de uma academia de ginástica. Revista Gestão Industrial, v. 8, n. 3, 2012.

BERGAMO, F.; GIULIANI, A.; GALLI, L. Modelo de lealdade e retenção de alunos para instituições do ensino superior: estudo teórico com base no marketing de relacionamento.

Brazilian Business Review, v. 8, n. 2, p. 43-66, 2011.

CLEMENTE-RICOLFE, J. Atributos relevantes de la calidad en el servicio y su influencia en el comportamiento postcompra. El caso de las hamburgueserías en España. Revista Innovar, v. 26, n. 62 , p. 69-78, 2016.

CRONBACH, L. Coefficient alpha and the internal structure of tests. Psychometrika, v. 16, n. 3, p. 297-334, 1951.

DA HORA, H.; MONTEIRO, G.; ARICA, J. Confiabilidade em questionários para qualidade: um estudo com o coeficiente Alfa de Cronbach. Produto \& Produção, v. 11, n. 2, p. 85-103, 2010.

DALMONECH, H.; GOULARTE, M.; RAMOS, P.; MONTE-MOR, D. Fatores que influenciam a indicação da instituição pública federal de ensino por seus alunos através do marketing boca a boca. Revista Eletrônica de Ciência Administrativa, v. 15, n. 1, p. 55, 2016.

DE ANDRADE, G.; DE ANDRADE, D.; MISAEL, G. Avaliação dos cursos de graduação à distância de uma universidade privada: um estudo quantitativo sob a perspectiva dos alunos. Ágora: Revista de Divulgação Científica, v. 19, n. 2, p. 4-23. 2015.

DE SOUZA, G.; DA COSTA G.; DA SILVA, M.; DAS NEVES B.; MIRA, V.; LEITE, M. Avaliação do curso de gerenciamento online na perspectiva dos egressos. Revista da Escola de Enfermagem da USP, v. 49, n. 2, p. 90-97, 2015.

DOS SANTOS, L.; CAMPOS, M.; DE LACERDA, C.; GOES, A. Desafios do ensino de libras na educação a distância. Comunicações, v. 22, n. 3, p. 203-219, 2015.

DOUGLAS, J.; DOUGLAS, A.; BARNES, B. Measuring student satisfaction at a UK university. Quality Assurance in Education, v. 14, n. 3, p. 251-267, 2006.

DUQUE E.; CERVERA T.; RODRÍGUEZ R. Estudio bibliométrico de los modelos de medición del concepto de calidad percibida del servicio en Internet. Revista Innovar, v. 16, n. 28, p. 223$243,2006$. 
ERGANG, S.; GODOY, L.; LORENZETT, D.; GODOY, T.; RIZZATTI, C.; GUARIENTI, E.; SILUK, J. Qualidade dos serviços em uma rede educacional: relação entre expectativa e percepção. Engevista, v. 14, n. 3, 2012.

GÓMEZ-REY, P.; BARBERA, E.; FERNÁNDEZ-NAVARRO, F. Measuring teachers and learners' perceptions of the quality of their online learning experience. Distance Education, v. 37, n. 2, p. 146-163, 2016.

GRUBER, T.; FUß, S.; VOSS, R.; GLÄSER-ZIKUDA, M. Examining student satisfaction with higher education services: using a new measurement tool. International Journal of Public Sector Management, v. 23, n. 2, p. 105-123, 2010.

HAIR, J.; ANDERSON, R.; BABIN, B.; BLACK, W. Multivariate data analysis. Upper Saddle River, NJ: Pearson, 2010.

INEP (2013). Censo da Educação Superior: Sinopses Estatísticas da Educação Superior: Graduação 2000-2012. Disponível em http://portal.inep.gov.br/sinopses-estatisticas-da-educacaosuperior. Acesso em 20 agosto, 2017.

LOURENÇO, C.; KNOP, M. Ensino superior em administração e percepção da qualidade de serviços: aplicação da escala SERVQUAL. Revista Brasileira de Gestão de Negócios, v. 13, n. 39, p. 219, 2011.

MALHOTRA, N. Marketing research: an applied orientation. Pearson Education India, 2008.

MANTOVANI, D.; GOUVÊA, M.; TAMASHIRO, H. Segmentação e qualidade em serviços educacionais: caso do curso de graduação a distância. Revista de Administração da Unimep, v. 13, n. 1, p. 91-116, 2015.

MILAN, G.; BRENTANO, J.; DE TONI, D. A qualidade percebida dos serviços prestados por agência de comunicação e a satisfação de clientes. Revista Brasileira de Gestão de Negócios, v. 10, n. 26, p. 17-26, 2008.

MILAN, G.; DE TONI, D.; MAIOLI, F. Atributos e dimensões relacionadas aos serviços prestados por instituição de ensino superior e a satisfação de alunos. Gestão \& Planejamento, v. 14, n. 2, 2013.

MOORE, M.; KEARSLEY, G. Educação a distância: uma visão integrada. São Paulo: Thomson Learning, 2007.

NETTO, C.; GIRAFFA, L. Avaliação da qualidade dos cursos de graduação a distância: o processo de acreditação como garantia de qualidade? RENOTE, v. 8, n. 1, 2010.

OROSCO, S.; PUGLIA, M.; DUARTE, K. Disciplina na modalidade semipresencial em enfermagem: avaliação de qualidade segundo sistema de indicadores. Revista de Enfermagem da UFSM, v. 4, n. 2, p. 265-275, 2014.

O'ROURKE, N.; HATCHER, L. A step-by-step approach to using SAS for factor analysis and structural equation modeling. Sas Institute, 2013. 
PINTO, S.; LAURINO, D.; LUNARDI, G. Processo de construção e validação de um instrumento de avaliação de cursos de graduação a distância. PRISMA.COM, n. 28, 2015.

PRIETO M.; DÍAZ M.; AGUILERA, I.; MONSERRAT S.; SANVICEN T.; SANTIAGO C.; CORELL A.; ÁLVAREZ-MON S. Nuevas combinaciones de aula inversa con just in time teaching y análisis de respuestas de los alumnos. Revista Iberoamericana de Educación a Distancia, v. 21, n. 1, 2018.

RADONS, D.; TORRES, C.; CERETTA, P. Mensuração da satisfação de clientes com serviços de fast food. Revista Eletrônica de Estratégia \& Negócios, v. 5, n. 3, p. 122-150, 2012.

SENTHILKUMAR, N.; ARULRAJ, A. SQM-HEI-determination of service quality measurement of higher education in India. Journal of Modelling in Management, v. 6, n. 1, p. 60-78, 2011.

ŠIMIĆ, M.; ČARAPIĆ, H. Education service quality of a business school: former and current students' evaluation. International Review on Public and Nonprofit Marketing, v. 5, n. 2, p. 181-191, 2008.

SONEHARA, W. Avaliação da qualidade de serviços em instituição de ensino superior utilizando os métodos SERVQUAL, SERVPERF e HEDPERF. 220p. Dissertação (Mestrado em Tecnologia) - Centro Estadual de Educação Tecnológica Paula Souza, São Paulo, SP, Brasil, 2011.

STREINER, D. Starting at the beginning: an introduction to coefficient alpha and internal consistency. Journal of Personality Assessment, v. 80, n. 1, p. 99-103, 2003.

SULTAN, P.; WONG, H. Antecedents and consequences of service quality in a higher education context: a qualitative research approach. Quality Assurance in Education, v. 21, n. 1, p. 70-95, 2013.

TOLENTINO, R.; GONÇALVES C.; TOLENTINO, R.; MONTEIRO, P. Avaliação da qualidade na educação a distância sob a perspectiva do aluno: proposição e teste de modelo usando equações estruturais. Revista de Gestão, v. 20, n. 3, p. 347-366, 2013.

TORRES M. Escala propuesta para medir la calidad del servicio de los cines. Revista Innovar, v. 20, n. 36, p. 157-171, 2010.

VEIGA, T.; MOURA, A.; GONÇALVES, C.; BARBOSA, F. O ensino a distância pela internet: conceito e proposta de avaliação. In Anais do XXII encontro da associação nacional dos programas de pós-graduação, São Paulo, 1998.

VENTURINI, J.; PEREIRA, B.; VIEIRA, K.; MILACH, F. Satisfação dos alunos do curso de Ciências Contábeis da UNIFRA: um estudo à luz das equações estruturais. In Anais do Congresso de Controladoria e Contabilidade da USP, São Paulo, 2008.

VIEIRA, M.; BARBOSA, T. A utilização das dimensões da qualidade em serviços como estabelecedora de parâmetros de avaliação no curso bacharelado em Administração na modalidade EAD. Administração Pública e Gestão Social, v. 3, n. 2, p. 243-256, 2012. 
ZEITHAML, V.; BERRY, L.; PARASURAMAN, A. Communication and control processes in the delivery of service quality. The Journal of Marketing, p. 35-48, 1988.

ZEITHAML, V.; BITNER, M.; GREMLER, D. Marketing de serviços: a empresa com foco no cliente. AMGH Editora, 2014. 